\title{
Semi-quantitative characterisation of binary salt mixtures with static secondary ion mass spectrometry (S-SIMS)
}

\author{
Rita Van Ham ${ }^{\mathrm{a}}$, Luc Van Vaeck ${ }^{\mathrm{a}}$, Annemie Adriaens ${ }^{\mathrm{b}, *}$, Freddy Adams ${ }^{\mathrm{a}}$, \\ Brittany Hodges ${ }^{c}$, Anthony Appelhans ${ }^{\mathrm{d}}$, Gary Groenewold ${ }^{\mathrm{d}}$ \\ ${ }^{a}$ University of Antwerp, Department of Chemistry, Universiteitsplein 1, B 2610 Antwerp, Belgium \\ ${ }^{\mathrm{b}}{ }_{\mathrm{C}}$ Ghent University, Department of Analytical Chemistry, Krijgslaan 281-S12, B 9000 Ghent, Belgium \\ ${ }^{\mathrm{c}}$ Purdue University, Department of Chemistry, 560 Oval Drive, West Lafayette, IN 47907-2084, USA \\ ${ }^{\mathrm{d}}$ Idaho National Engineering and Environmental Laboratory, Post Box 1625, Idaho Falls, ID 83415-2208, USA
}

Received 14 July 2005; accepted 28 July 2005

Available online 3 October 2005

\begin{abstract}
The use of static secondary ion mass spectrometry for the semi-quantitative characterisation of major components by means of molecule specific ion signals has been investigated for binary salts. The calibration of the peak intensities as a function of the local concentration has been based on reference mixtures. The preparation of the mixtures has been proven to be a major bottleneck. Five different approaches have been tried out. Fast freezing with subsequent cryo-lyophylisation is the preferred method to obtain adequate molecular mixtures for the empirical calibration of relative peak intensities in S-SIMS as a function of the local concentration. The applicability has been verified in two different laboratories and three instrumental S-SIMS configurations. Monoatomic as well as polyatomic primary ions have been used. Specifically, comparative work involved the use of $\mathrm{Ga}^{+}$primary ions in a time-of-flight (TOF) S-SIMS and $\mathrm{ReO}_{4}{ }^{-}$polyatomic projectiles in an ion trap and a triple quadrupole instrument, used in the single mass selection mode. In all cases, the local content can be determined with an experimental uncertainty of 10-20\% using the molecular (adduct) ion signal intensities in either the positive or negative ion detection mode. (C) 2005 Elsevier B.V. All rights reserved.
\end{abstract}

Keywords: Static-SIMS; Quantification; Mixtures; Binary salts; Polyatomic; Sample preparation

\section{Introduction}

Methods such as static secondary ion mass spectrometry (S-SIMS) or laser microprobe mass spectrometry (LMMS) are increasingly appreciated for their capability to generate direct information on the molecular composition of inorganic and organic analytes at the surface of solids with high lateral resolution and depth resolution [1-5].

However, their reputation with respect to quantitative analysis is less positive [6]. An important reason for this is the complication by matrix effects that hamper all solid-state ionisation methods. Conventional mass spectrometry with the ionisation of analytes in the gas phase is inherently better

\footnotetext{
* Corresponding author. Tel.: +32 9264 4826; fax: +32 92644960

E-mail address: annemie.adriaens@ugent.be (A. Adriaens).
}

suited to quantitative determinations as the ionisation and subsequent fragmentation is unimolecular in nature, unaffected by the other components of the sample. Hence, the ion yield and the instrumental effects (transmission, detection efficiency as a function of $\mathrm{m} / \mathrm{z}$ ) can be accounted for by simple calibration samples. In contrast, direct ionisation of solids inevitably implies that all species within the micro volume where the initial energy is deposited, can influence the desorption and ionisation of the analyte. Whereas matrix effects can be well controlled for trace analytes in a matrix with almost homogeneous composition (e.g., doped semiconductor materials in SIMS), the problem aggravates in the case of major component analysis. A second barrier for quantitative S-SIMS is the generation of molecular information from a volume as thin as a single monolayer. The lack of independent methods to verify the composition of calibration mixtures at 
the same level of information and information depth makes preparation of reference samples a major bottleneck.

The purpose of this paper is to report on a first feasibility study to use the molecular (adduct) ion signals in S-SIMS for the quantitative characterisation of inorganic salts present as major components in the surface layer of micro-objects. Five methods for the preparation of suitable calibration mixtures have been tried out. Specifically, crushing solids combined with pressing into pellets or with cascade impactor sampling from a fluidised bed, aerosol spraying and cascade impactor collection of solutions, fast freezing and subsequent cryolyophilisation as well as melts of binary salts have been investigated. The cryo-technique has been found the method of choice to make suitable reference mixtures and its applicability has been verified in two laboratories using three different S-SIMS instrumental configurations with mono- or poly-atomic primary ions. Specifically, $\mathrm{Ga}^{+}$bombardment has been applied in a TOF S-SIMS whereas $\mathrm{ReO}_{4}{ }^{-}$projectiles have been used in conjunction with experimental ion trap (IT) S-SIMS as well as with triple quadrupole (TQ) instrument, used in the single $\mathrm{m} / \mathrm{z}$ filtering mode. The empirical calibration of the intensity ratios of specific ions as a function of the local concentration has been used to estimate the experimental uncertainty in the semi-quantitative characterisation of binary salt mixtures.

\section{Experimental}

\subsection{Instrumental}

TOF S-SIMS analyses were performed the University of Antwerp with an ION TOF IV instrument (Cameca, France). The $\mathrm{Ga}^{+}$gun was operated at a beam voltage of $25 \mathrm{kV}$ in the bunched mode, yielding pulses of $20 \mathrm{~ns}$. Mass resolution was sufficient for baseline separation of nominal masses up to $\mathrm{m} / \mathrm{z}$ 1000. Secondary ions from $3 \times 10^{6}$ pulses were accumulated in $300 \mathrm{~s}$. The analysed area was $300 \mu \mathrm{m} \times 300 \mu \mathrm{m}$ and the total ion dose was less than $10^{11}$ ions $\mathrm{cm}^{-2}$. Electron flooding was applied for charge compensation.

The TQ S-SIMS used in this study was converted at INEEL from a commercial Extrel instrument (Pittsburgh, PA). Perrhenate $\left(\mathrm{ReO}_{4}{ }^{-}\right)$primary ions were generated by heating a ceramic powder of alkali earth perrhenate blend with e.g., $\mathrm{Eu}_{2} \mathrm{O}_{3}$ in a rhenium tube at about $1000 \mathrm{~K}$. The source, developed at INEEL, was operated in the continuous mode on the TQ S-SIMS and in the pulsed mode on the IT S-SIMS [7]. The $5.5 \mathrm{keV}$ primary ions impacted on the sample with an incidence angle of $35^{\circ}$. A current of typically $180 \mathrm{pA}$ was delivered over a spot with a diameter of $2.5 \mathrm{~mm}$. The TQ SSIMS was scanned from $m / z 5$ to 400 in $300 \mathrm{~s}$. The sequential mass analysis implied that secondary ions detected at individual $\mathrm{m} / \mathrm{z}$ were generated by less than $4 \times 10^{10}$ ions $\mathrm{cm}^{-2}$. Combination of $\mathrm{ReO}_{4}{ }^{-}$primary ions with an alternating extraction field eliminated charge build-up in non-conducting samples.
A commercial Finnigan ITMS (San Jose, CA) was converted at INEEL for S-SIMS by drilling holes through the centre of the caps, allowing the primary beam to be directed perpendicularly on the exposed sample surface at the opposite side of the IT. A $\mathrm{ReO}_{4}{ }^{-}$gun bombarded the sample randomly over an area with diameter of $2.5 \mathrm{~mm}$ with a beam current of $570 \mathrm{pA}$ (DC). The duration of the primary ion pulse was varied between 4 and $60 \mathrm{~ms}$, yielding an ion dose between $10^{11}$ and $2 \times 10^{12}$ ions $\mathrm{cm}^{-2}$. The IT was operated in the mass instability mode. Secondary ions were consecutively driven through the primary beam hole in the end cap, deflected and detected by an electron multiplier. Positive ions were analysed using a buffer gas pressure of $3.6 \times$ $10^{-6}$ mbar, ionisation time of $4 \mathrm{~ms}$, low mass cut-off of 40 , and a leakage ring voltage of $21 \mathrm{~V}$. Negative ions required settings of $2.7 \times 10^{-6} \mathrm{mbar}, 60 \mathrm{~ms}, 50$ and $-60 \mathrm{~V}$, respectively. No ejection pulses were applied. The $\mathrm{m} / \mathrm{z}$ range was scanned up to $m / z 600$. Each spectrum was summed from 245 scans.

\subsection{Sample preparation}

Five methods for the preparation of suitable calibration mixtures were tried out.

Mixtures of binary salts were ground in a mortar and pressed into pellets (diameter $1 \mathrm{~cm}$, thickness $1-2 \mathrm{~mm}$ ), using a common dial at $6 \mathrm{tcm}^{-2}$. Only a piece of about $1 \mathrm{~mm} \times 1 \mathrm{~mm}$ with a flat surface was mounted in the TOF S-SIMS sample holder allowing the instrument to be pumped down within a reasonable time.

Alternatively, the crushed powder was suspended in a vertical stream of nitrogen and the $1-5 \mu \mathrm{m}$ fraction was collected with a Berner cascade impactor (flow rate $26 \mathrm{~L} \mathrm{~min}^{-1}$ ). The impaction plates were modified to allow silicon wafer to be used as collection substrates and mounted flush with the original impaction surface [8].

As a third sample preparation, solutions of about $0.2 \mathrm{~g}$ of salts in 50\% methanol/water $(\mathrm{v} / \mathrm{v})$ were sprayed with an airbrush (Badger, IL) under a pressure of about 1 bar, adjusted for a spray diameter of $1 \mathrm{~cm}$ at a distance of $5 \mathrm{~cm}$. The microdroplets were dried by transfer through a tube (length $40 \mathrm{~cm}$, diameter $1.5 \mathrm{~cm}$ ) under a nitrogen stream of $26 \mathrm{~L} \mathrm{~min}^{-1}$. Particles of 2-4 $\mathrm{m}$ were collected for about $10 \mathrm{~min}$ with a Berner cascade impactor using silicon wafer collection substrates.

Cryo-samples for TOF S-SIMS were prepared by spotting $10 \mu \mathrm{L}$ of aqueous salt solutions (concentration in the range of $5 \mathrm{~g} \mathrm{~L}^{-1}$ ) on "polished" stainless steel TOF S-SIMS sample holder, to ensure reproducible spreading of the solution on the surface. Fast freezing was achieved by putting the sample holders on the platform, pre-cooled during $30 \mathrm{~min}$ with liquid nitrogen, of the in-house developed cryo-lyophilisation device, described before [9]. The vacuum chamber was pumped down immediately to a pressure of $10^{-3}$ mbar. The external cold finger connected to the platform was left in contact with liquid nitrogen for about 10-15 min and subsequently with molten ice for $3 \mathrm{~h}$. Final warming up to room 
temperature occurred by ambient air. An adequate restriction in the venting line was needed to break the vacuum and avoid blow off of the freeze-dried material. The packing and surface roughness of the sample was improved by gently pressing the material with a thin glass plate. Samples were kept in vacuum until TOF S-SIMS analysis, no longer than $24 \mathrm{~h}$ after preparation.

The preparation of freeze-dried mixtures for IT and TQ S-SIMS required modification of the procedure described because of the specific sample holders to be used. A polished steel nail head with a diameter of $1.25 \mathrm{~mm}$ was used as initial temporary substrate. After spotting $5 \mu \mathrm{L}$ of an aqueous salt, the nail was put in a dedicated holder, immersed in liquid nitrogen, to induce fast freezing. Cryo-lyophilisation was achieved at a pressure of $10^{-6}$ mbar in about $12 \mathrm{~h}$ without additional cooling. The vertical mounting of the sample surface in IT and TQ S-SIMS required transfer of the microcrystals from the temporary sample holder to a steel nail (for IT) or flattened wire (for TQ), covered with double-sided tape.

Melts were prepared by heating mixtures of crushed powders in a porcelain crucible at $800^{\circ} \mathrm{C}$ for $12 \mathrm{~h}$. Subsequent cooling to room temperature was achieved in about $12 \mathrm{~h}$. The resulting solid was ground again and pressed into pellets.

\section{Results and discussion}

\subsection{Preparation of calibration mixtures}

The requirement to preserve the structure and molecular composition of the analyte excludes the use of several currently used methods to prepare reference samples, e.g., thermal evaporation, ion implantation, plasma deposition, etc. As a result the ideal situation of different analytes that are "perfectly" mixed at the level of the molecular lattice, are is in practice almost impossible to attain, except in very few cases, such as Langmuir-Blodgett layers for organic compounds. However, in practical micro-analytical applications, it is sufficient that each individual micro-volume has a reproducible composition. The collision cascade under $25 \mathrm{keV} \mathrm{Ga}^{+}$impact is believed to extend over an area with a diameter of $10 \mathrm{~nm}$ around the impact point and over a distance of up to $50 \mathrm{~nm}$ in depth. Whenever the mixtures used for calibration contain compositional discontinuities (laterally or in depth), verification of the molecular composition within the micro volume of interest for S-SIMS is impossible because no independent analytical method exist that is capable of delivering molecular information on a similar scale. The purpose of this section is to discuss the adequacy of five methods tried out for the preparation of molecular mixtures that can be used for the empirical calibration of S-SIMS.

Dry mixing of crushed powder looks an interesting approach, being fast, simple, applicable to compounds regardless their solubility and stability in solution (e.g., oxides) with a minimal risk of contamination. However, the practical limit of final grain sizes is in the range of one to several micrometers. Neither pressing of the resulting material into pellets nor suspension in a fluidised bed and sampling with a cascade impactor alleviates the problem, because each grain has dimensions far above the interaction or information volume of S-SIMS and contains only one component. Incomplete surface coverage causes additional variability.

Although inapplicable to e.g., oxides, solutions offer the advantage of starting with a perfectly homogeneous mixture but require adequate means to avoid the separation of the analytes in distinct solid phases during crystal formation, in particular when the solvent evaporates slowly. Fast evaporation creates the problem that the separation of analytes during the crystallisation from solution is governed by kinetic and thermodynamic factors, none of which are readily predicted from available data. As a result, the final composition of a residue from the rapid evaporation of an equimolar $\mathrm{NaBr}-\mathrm{KCl}$ solution is hard to predict accurately. Hence, this study has been confined to binary mixtures of salts with a common anion.

The only approach left when starting from solutions is empirical in nature and aims at micro crystals, which are sufficiently small in comparison to the dimensions of the volume of interest for analysis. Fast evaporation and/or limitation of the amount of solution, from which the solid residue is made, have led to the cascade impactor sampling of nebulised solutions. Although the airbrush-drying tube set-up is designed to keep the micrometer size micro droplets separated before impact, it turns out that complete evaporation of the solvent is hard to achieve and significant recrystallisation in the impacted still wet material.

Ultra-fast freezing and subsequent slow evaporation of the solvent at low temperature in vacuum (cryo-lyophilisation) is known to preserve the distribution of e.g., mobile cations in tissues [10]. The freezing must be instantaneous to prevent phase separation whenever sufficient amounts of solution are enclosed in the initially formed compartments. The lyophilisation at low temperature and in vacuum aims at removal of "individual" solvent in such a way that never a micro-film or -droplet of solution is formed at the surface.

According to our experience, the cryo-approach reveals to be the method of choice to make reference mixtures for the calibration of micro-analytical methods such as S-SIMS and FT LMMS [9]. Furthermore, microscopic observation of the sample provides an easy way to guide the optimisation of the sample preparation. The secondary electron images in Fig. 1 shows the formation of giant particles when a commercial cryo-set-up with a cooling capability limited to $-5^{\circ} \mathrm{C}$ has been used. The corresponding secondary ion images evidence the enrichment of $\mathrm{Br}^{-}$and $\mathrm{I}^{-}$in distinct zones. The electron micrographs in the lower part of Fig. 1 shows the improvement resulting from the use of a dedicated cryo-device. Under not yet fully optimised temperature and timing conditions, a still heterogeneous size distribution of the crystals and presence of cubic structures as large as several tens of micrometer is observed. However, tuning the conditions allows samples to 

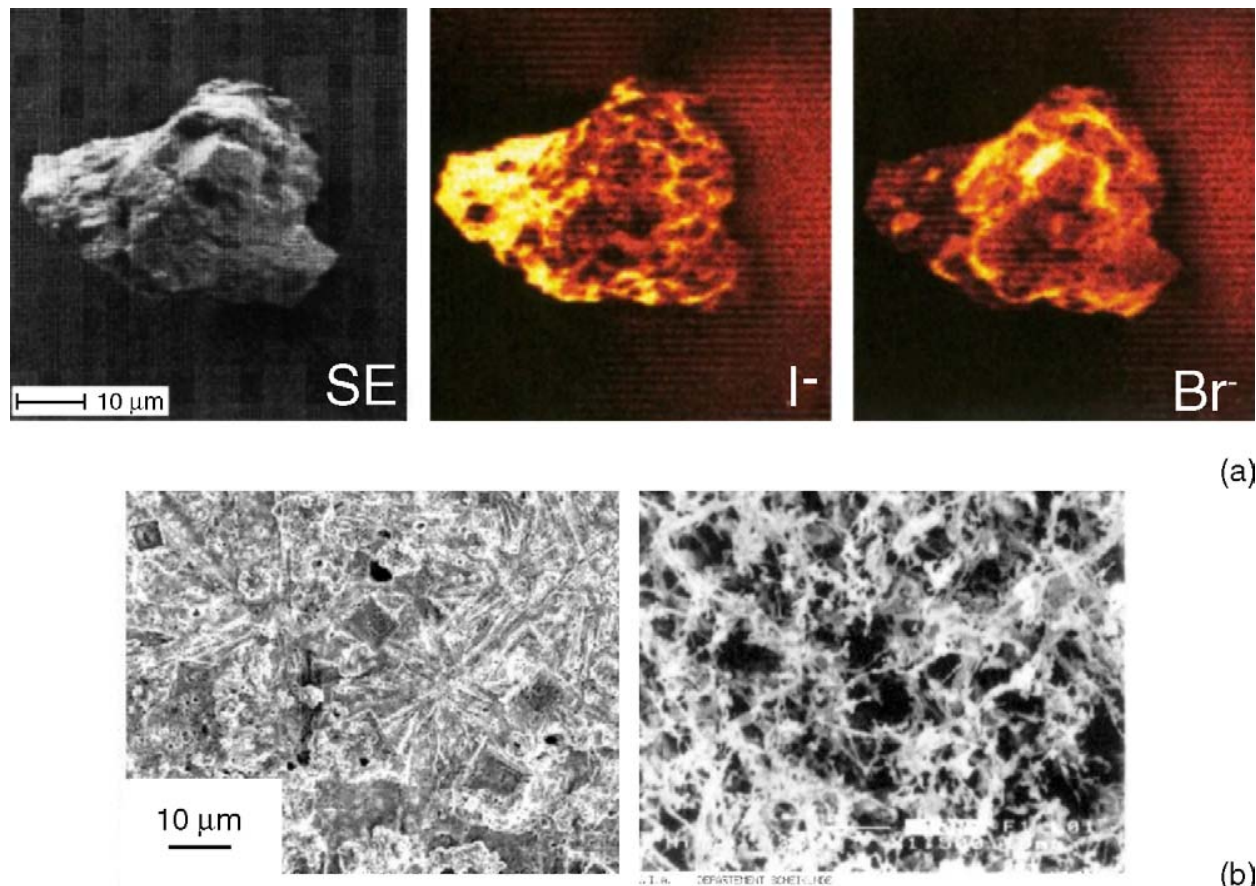

(a)

(b)

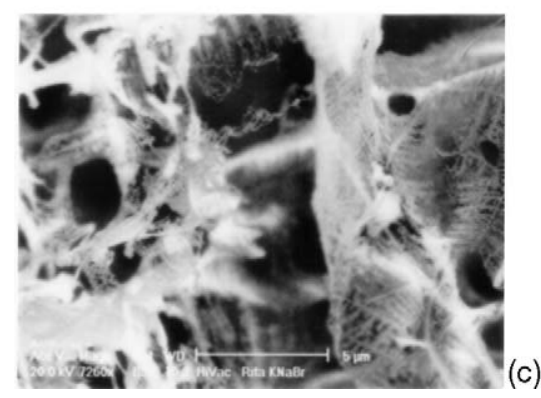

Fig. 1. Formation of large microcrystals and local enrichment by inadequate lyophilisation of $\mathrm{Nal}-\mathrm{NaBr}$ mixtures, (a) left: secondary electron image; middle: ion image for $\mathrm{I}^{-}$; right: ion image for $\mathrm{Br}^{-}$. (b) Secondary electron image of an unsatisfactory (left) and a good (right) freeze-dried $\mathrm{NaBr}-\mathrm{KBr}$ mixture prepared in Antwerp, Belgium. (c) Secondary electron image of a good freeze-dried mixture prepared in Idaho Falls, Idaho, USA.

be obtained with sponge-like arrangement of micro-crystals, each of which is less than $0.5 \mu \mathrm{m}$, arranged in a network of fine needles. The ultimately thin filaments of the samples prepared at INEEL reflect the improvement of the fast freezing step. The extremely dry environment in Idaho in comparison to Belgium has indeed allowed the initial solution to be spotted on a pre-cooled sample holder. However, S-SIMS data have revealed that both types of cryo-samples are adequate as empirical calibration mixtures. The in-sample and between-sample variability remains within the experimental error of instrumental analysis.

Melts of binary salts have been tried out as a final method, limited however to thermally stable analytes with similar melting temperatures and lattices that allow cations or anions to be interchanged during crystal growth. This approach has been successfully used by Honda et al. [11] for the investigation of ion formation from $\mathrm{CsCl}-\mathrm{KCl}$ mixtures. Our experiments on the $\mathrm{NaBr}-\mathrm{KBr}$ systems have shown that the use of melts is equally adequate or slightly better than the cryo-method for the preparation of calibration samples [9].

\subsection{Qualitative information}

The first step in trying to quantify is the selection of the ions, which maximise intensity as well as molecular specificity. As to the former aspect, it is practically required that the corresponding peak contains at least 5000 counts after $300 \mathrm{~s}$ analysis time for the sample with the lowest concentration. For both methods, TQ S-SIMS and IT, the threshold is set at 50 cpsat. Molecular specificity requires that molecular ions or adducts are used. The risk of isobaric interference due to e.g., contaminants decreases with increasing $\mathrm{m} / \mathrm{z}$.

The mass spectra of pure alkali halides under monoatomic and polyatomic primary ion bombardment have been reported before [12]. Fig. 2 shows the TOF-SIMS data from 

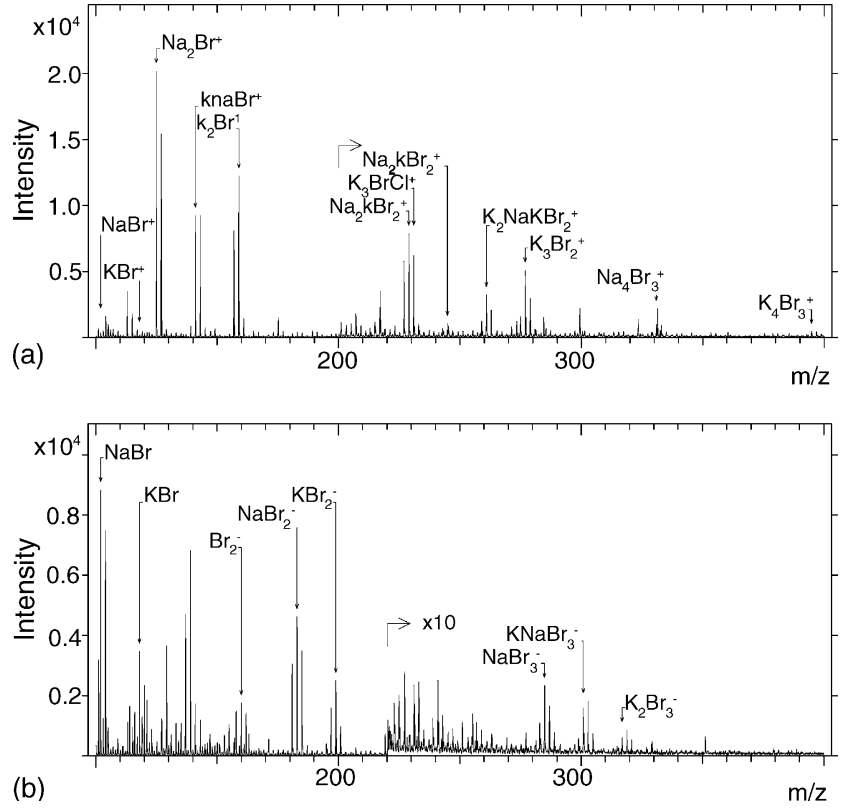

Fig. 2. Positive (a) and negative (b) ion mass spectra of a freeze-dried mixture containing $46 \mathrm{~mol} \%$ of $\mathrm{NaBr}$ and $9 \mathrm{~mol} \% \mathrm{NaBr}$, respectively.

freeze-dried 46 and 9 mol\% mixtures. Although atomic ions account for typically over $98 \%$ of the total ion current (TIC) in TOF S-SIMS, molecule-specific adduct ions still yield prominent peaks clearly distinguishable from the background. Signals from adducts of $\mathrm{Na}^{+}$or $\mathrm{K}^{+}$to one or several $\mathrm{NaBr}$ or $\mathrm{KBr}$ neutrals show "exponentially" decaying intensities with increasing number of neutrals in the ion. Of particular interest are the "mixed" adducts, containing structural units from both analytes, e.g., $\mathrm{KBr} \cdot \mathrm{Na}^{+}$or $\mathrm{NaBr} \cdot \mathrm{K}^{+}$. The negative ion mass spectra contain strong signals from molecular ions and monomeric adducts but their absolute intensity stays a factor of typically 10 under that of the monomeric adducts in the positive ion detection mode. The smallest anion that contains a contribution from both analytes is the dimeric adduct ion, i.e., $\mathrm{NaBr} \cdot \mathrm{KBr} \cdot \mathrm{Br}^{-}$(as opposed to the monomeric adducts in the positive ion detection mode). Summarising, the monomeric adducts and the molecular anions are the ions of choice for the quantitative characterisation.

The mass spectra in Fig. 3, recorded with TOF S-SIMS from the melts, show significantly higher absolute intensities for the major ions in comparison to the cryo-samples. The ion yield improvement is typical for pellet samples relative to closed particle layers. However, no significant differences in the relative intensities in mass spectra from pellets or particles occur. Additional peaks are related to the presence of lithium salts, most likely introduced by the use of the porcelain crucibles.

The mass spectra in Fig. 4, acquired from a 50 mol\% mixture with $\mathrm{ReO}_{4}{ }^{-}$polyatomic projectiles and TQ S-SIMS, show a significantly reduced contribution of atomic ions to the TIC in comparison to TOF S-SIMS with $\mathrm{Ga}^{+}$primary ions. For instance, the peak intensity ratio of $\mathrm{Na}^{+} / \mathrm{NaBr} \cdot \mathrm{Na}^{+}$
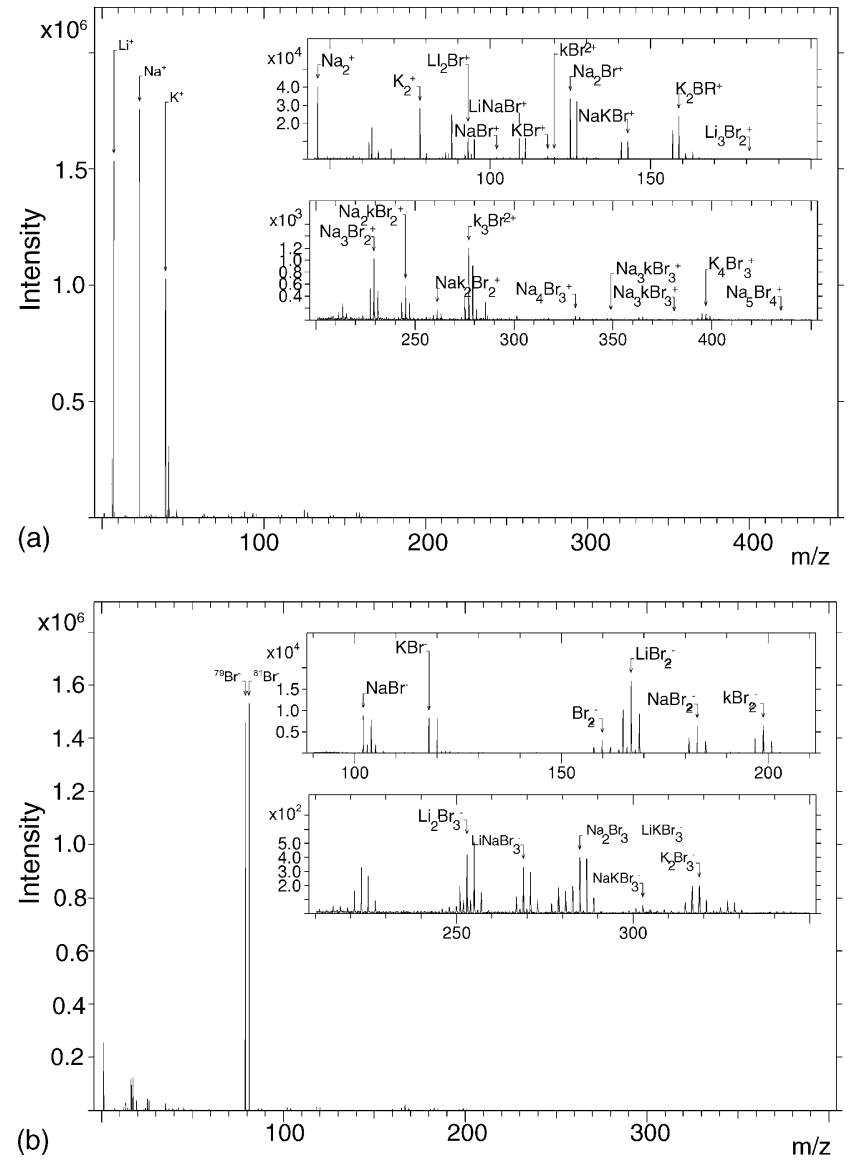

Fig. 3. Positive (a) and negative (b) ion mass spectra of a melted $\mathrm{NaBr}-\mathrm{KBr}$ mixture $(49 \mathrm{~mol} \% \mathrm{NaBr}$ ) contaminated with $\mathrm{Li}$.

in the data from pure analytes is typically 7 and 300 in TQ SSIMS with $\mathrm{ReO}_{4}{ }^{-}$and TOF S-SIMS with $\mathrm{Ga}^{+}$, respectively. A similar observation applies to the negative ion mode. The ratios obtained with $\mathrm{SF}_{5}{ }^{+}$projectiles in TOF S-SIMS are similar to the ones recorded with $\mathrm{ReO}_{4}{ }^{-}$in TQ S-SIMS. Again, the monomeric adducts (positive and negative) as well as the molecular anions remain the best choice to base upon the semi-quantitative characterisation of mixtures.

The combination of $\mathrm{ReO}_{4}{ }^{-}$polyatomic bombardment with an IT MS amplifies the trend already seen in TQ S-SIMS. Specifically, relative intensities of the high $\mathrm{m} / \mathrm{z}$ adducts significantly increase in comparison to the ion transport analysers. Positive adduct ions with one up to four neutral molecules are detected within an intensity range of less than one decade. The difference between data from IT and TQ S-SIMS, using the same ion gun, can be explained by the extended time domain of the mass analysis in the IT instrument. Ions are typically trapped for over 1-100 ms whereas detection in the ion transport mass spectrometry occurs within less than $500 \mu \mathrm{s}$. As a result, only the most stable ions (lowest internal energy) survive in IT S-SIMS. The same line of reasoning explains the low intensity of molecular radical anions relative to that of the more stable even-electron species when IT S-SIMS is compared to TQ and TOF S-SIMS. 

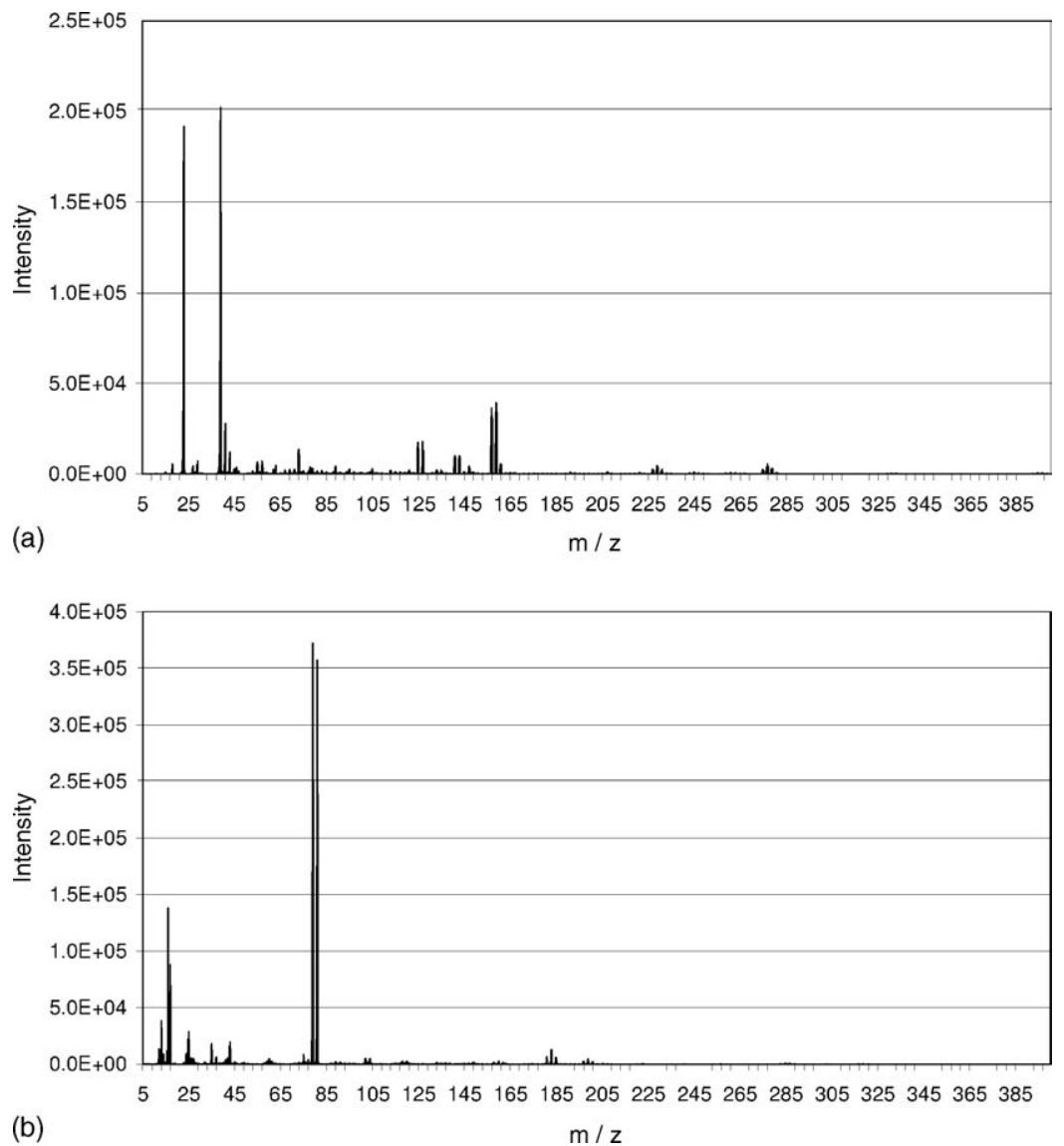

Fig. 4. Positive (a) and negative (b) ion mass spectra of a freeze-dried mixture containing $50 \mathrm{~mol} \%$ of $\mathrm{NaBr}$ recorded with Q S-SIMS.

\subsection{Calibration of TOF S-SIMS with freeze-dried mixtures}

The variability of the TIC practically prevents the use of absolute intensities as a direct measure for the local concentration. One of the main reasons is the huge intensity difference between the atomic and molecule-specific ions. The former account for up to $98 \%$ of the TIC and therefore, limitation of one ion per $\mathrm{m} / \mathrm{z}$ and per ionisation cycle is readily exceeded under conditions that allow detection of high $\mathrm{m} / \mathrm{z}$ ions with sufficient intensity in a reasonable analysis time. Although the counting errors on the atomic ion signals cause significant variability of the TIC values, relative intensities of higher $\mathrm{m} / \mathrm{z}$ ions show acceptable reproducibility. The insample and between-sample variability is typically confined to a relative standard deviation (R.S.D.) of $2-10 \%$, although outliers occasionally occur. Because the reproducibility of relative intensities in mixtures approaches those observed for pure analytes, sample homogeneity is considered to be adequate in a first approximation.

Fig. 5 shows the empirical calibration of the intensity ratios of selected molecule-specific ions as a function of the molar content in the initially used solutions. Because of the high precision of isotope ratio measurements (2\%) [13], the peak area of only one signal within an isotopic cluster is adequate. The intensity ratio $\mathrm{Na}_{2} \mathrm{Br}^{+} / \mathrm{K}_{2} \mathrm{Br}^{+}$as a function of the local $\mathrm{NaBr}$ content data can be reasonably fitted with a continuous but non-linear function. This type of relationship between the peak intensity ratio and concentration is inherently related to the analysis of major components and the way of representation and does not necessarily reflect anomalies in the surface composition or ionisation. Specifically, the smooth and continuous variation of the adduct intensity ratios with the $\mathrm{NaBr}$ content allows an at least empirical calibration to be used for the semi-quantitative characterisation of binary mixtures by means of molecule-specific signals. From the typical precision of the adduct ratio (i.e., 10\%), the uncertainty on the local concentration derived from the calibration of the positive ions is assessed to be within 10-20\% for a molar $\mathrm{NaBr}$ content of 56 and $77 \mathrm{~mol} \%$, respectively. For the negative ions, the corresponding value lies between 2 and $15 \%$.

Strikingly, the $\mathrm{NaKBr}^{+} / \mathrm{K}_{2} \mathrm{Br}^{+}$intensity ratio has been found to exhibit no useful dependence on the local $\mathrm{NaBr}$ content in freeze-dried mixtures. According to our opinion, this can be due to the fact that the sample primarily contains crystallites of pure $\mathrm{NaBr}$ or $\mathrm{KBr}$ with dimensions above the volume, affected by a single primary ion interaction. In that 

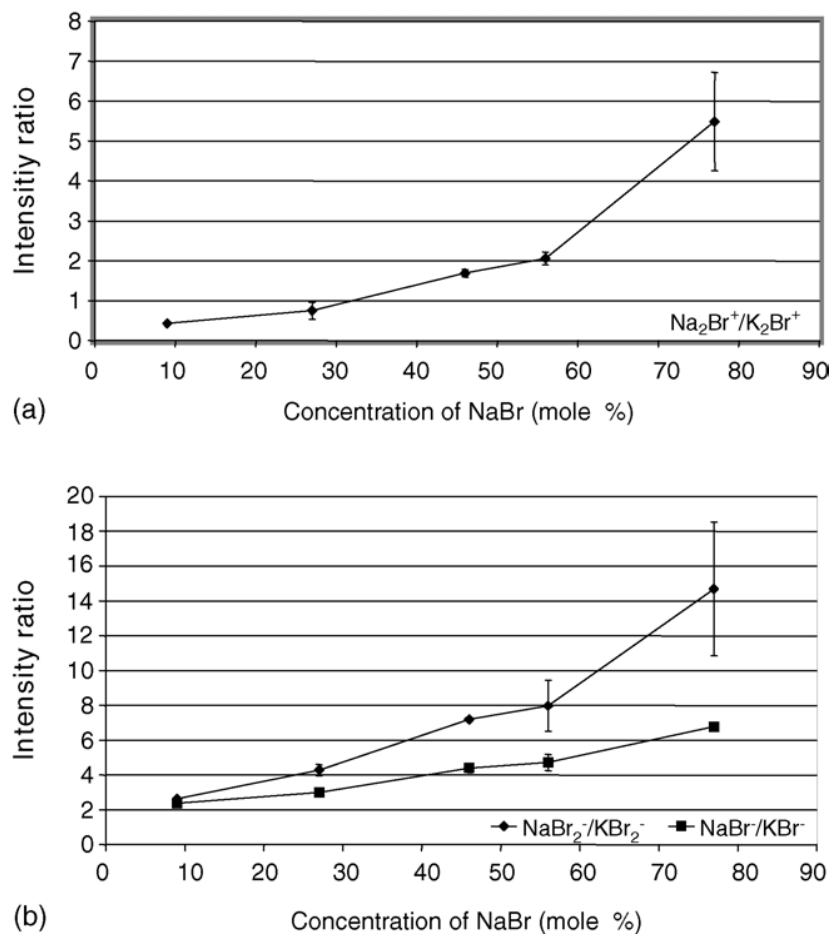

Fig. 5. Calibration of the adduct intensity ratios of $\mathrm{Na}_{2} \mathrm{Br}^{+} / \mathrm{K}_{2} \mathrm{Br}^{+}$(a) and $\mathrm{NaBr}_{2}{ }^{-} / \mathrm{KBr}_{2}{ }^{-}$(b), $\mathrm{NaBr}^{-} / \mathrm{KBr}^{-}$(b) as a function of the $\mathrm{NaBr}$ concentration in a $\mathrm{NaBr}-\mathrm{KBr}$ mixture using freeze-dried samples.

case, the probability of generating $\mathrm{KNaBr}^{+}$ions is directly proportional to the length of the interfaces between microcrystals of different composition.

It can be interesting to compare the latter results with a parallel study with FT LMMS using similar freeze-dried mixtures [3]. The $\mathrm{KNaBr}^{+}$adducts have been used without problems for semi-quantitative calibration, as opposed to TOF S-SIMS. In FT LMMS, over 100 individual microcrystals within an area with a diameter of $5 \mu \mathrm{m}$ are bombarded with many photons during about $10 \mathrm{~ns}$. In principle, all ions needed for detection are generated in a single pulse. This causes the desorption and selvedge ionisation processes to be collective in nature and less dependent on the presence of perfectly mixed lattices or of separated phases with different composition.

\subsection{Calibration of TOF S-SIMS with molten mixtures}

The data in Fig. 6 supports the tentative explanation in the previous section. In contrast to the use of freeze-dried reference samples, no significant difference is seen between the calibration by ions containing components from both analytes and that of ions due to only one analyte. Unlike the case of freeze-dried mixtures, the peak intensity ratio $\mathrm{KNaBr}^{+} / \mathrm{K}_{2} \mathrm{Br}^{+}$can be used equally well as that of $\mathrm{Na}_{2} \mathrm{Br}^{+} / \mathrm{K}_{2} \mathrm{Br}^{+}$. This observation suggests a more intimate or molecular mixing of the components in molten mixtures than in freeze-dried samples. Apparently, the substantial lithium contamination does not disturb the experiment, most likely
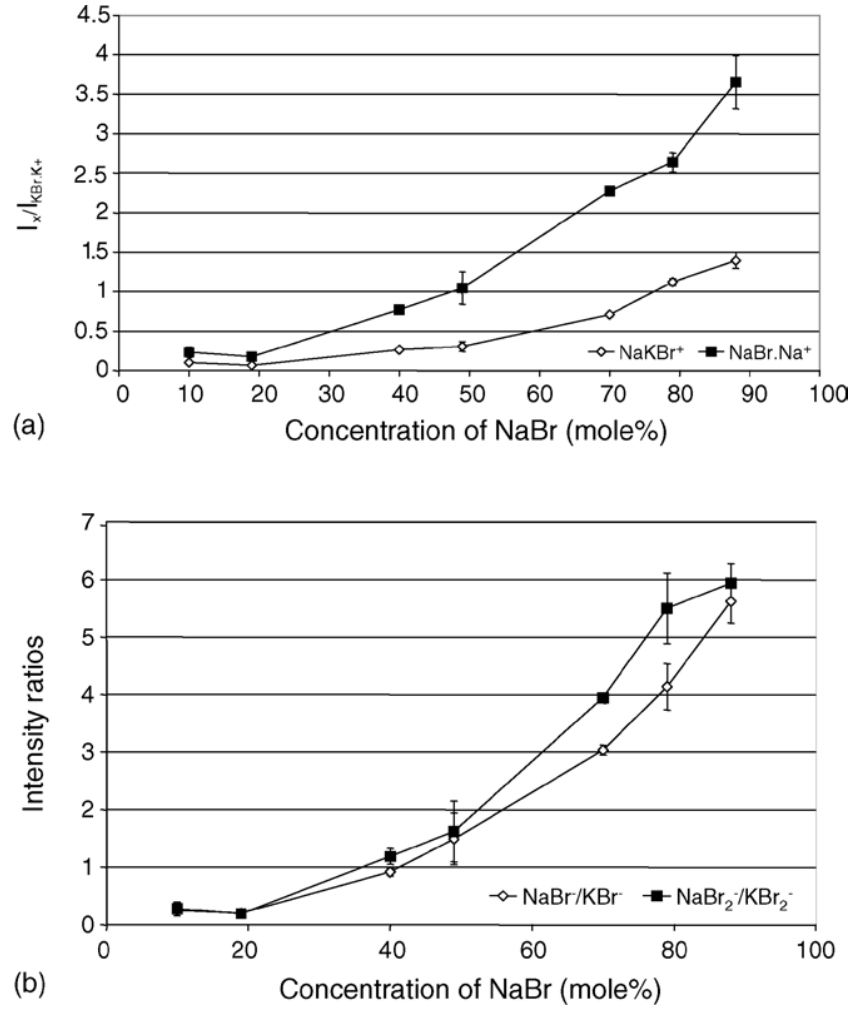

Fig. 6. Calibration of specific positive (a) and negative (b) ion intensity ratios as a function of the $\mathrm{NaBr}$ concentration in a $\mathrm{NaBr}-\mathrm{KBr}$ mixture using molten samples recorded with TOF S-SIMS.

because of its random distribution throughout the lattice. The precision of $2-10 \%$ for the peak intensity ratios in the positive ion mode brings the uncertainty on the measurement of the concentration around $10 \%$.

Fig. 7 shows the negative adduct ion intensity ratios plotted as a function of the variable $X=C /(100-C)$ where $C$ is the concentration of $\mathrm{NaBr}$ in mol\%. This relationship is physically unexpected. Specifically, two types of functions can be anticipated. On the one hand, there is the obvious linear dependence the molecular (adduct) intensity ratio on the normalised variable $X$ (giving some kind of "exponential" in Fig. 5). This means that the signal intensities simply reflect (1) the availability and uniform ionisation of both analytes at the surface of the samples and (2) that no significant surface depletion of enrichment has taken place so that the initial bulk concentration can be used. On the other hand, whenever selvedge ionisation prevails, it is conceivable that the $Y$ varies with $X$ according to a second order dependence.

\subsection{Calibration of TQ S-SIMS with freeze-dried mixtures}

Fig. 8 depicts the empirical calibration of the signal intensities of positive and negative ions ratios as a function of the converted variable $X$. An essentially linear relationship is found for positive ions as opposed to the inverted parabola seen in TOF S-SIMS. According to our opinion, the differ- 


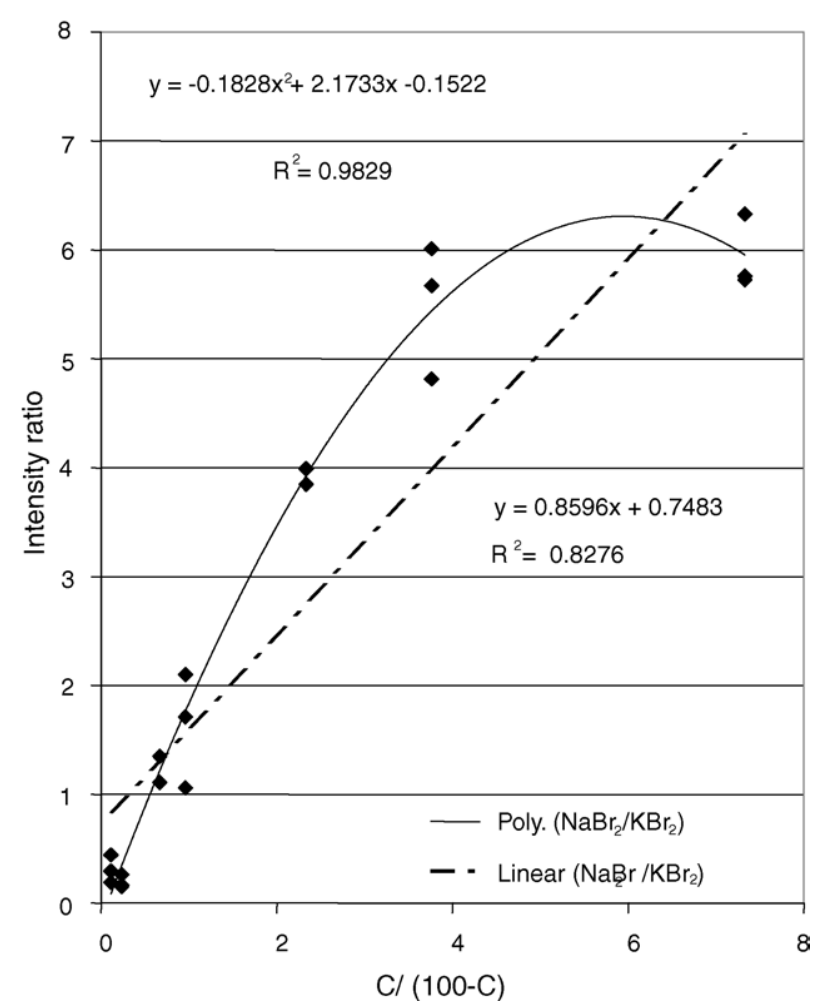

Fig. 7. Calibration of specific negative ion intensity ratios as a function of the converted variable $X=(C /(100-C))$ with $C=$ molar concentration of $\mathrm{NaBr}$ in a $\mathrm{NaBr}-\mathrm{KBr}$ mixture recorded with TOF S-SIMS.
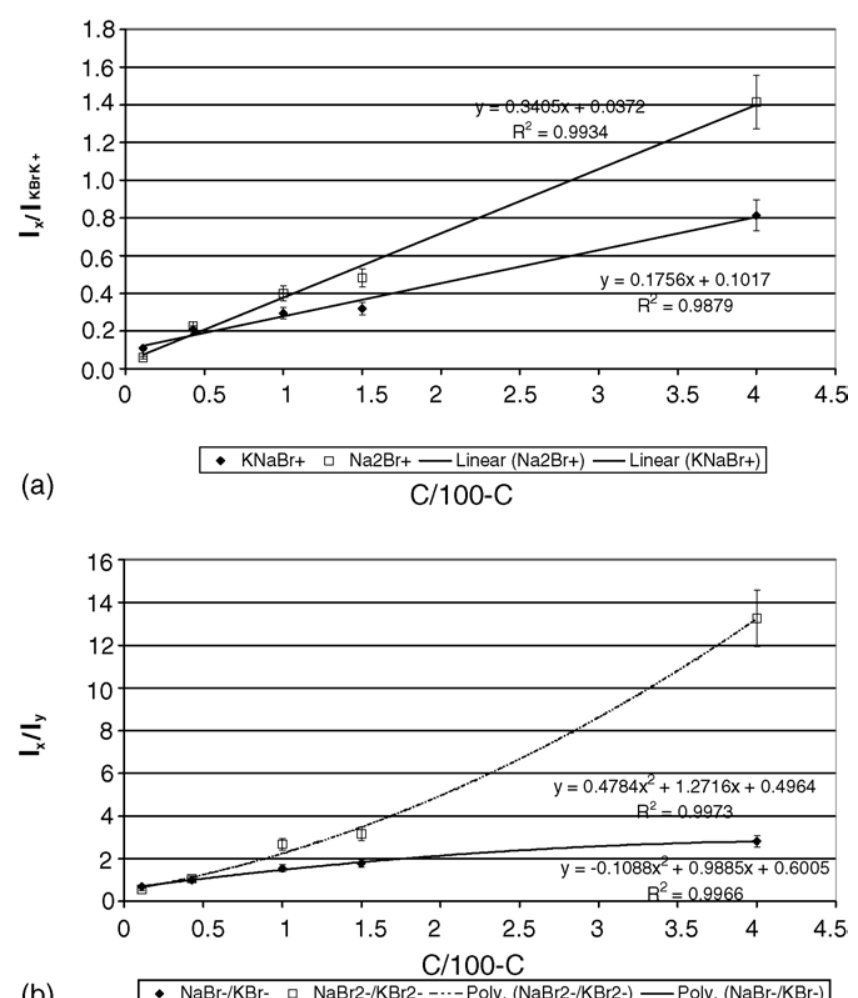

(b)

Fig. 8. Calibration of specific positive (a) and negative (b) ion intensity ratios as a function of the $\mathrm{NaBr}$ concentration in a $\mathrm{NaBr}-\mathrm{KBr}$ mixture using freeze-dried samples recorded with Q S-SIMS. ence between the two different sets of results relates to the use of a defocused beam, allowing the mixture to be randomly sampled over a larger area than in TOF S-SIMS.

As to the cations, the peak intensities of $\mathrm{NaBr} \cdot \mathrm{Na}^{+}$and $\mathrm{NaBr} \cdot \mathrm{K}^{+}$normalised to that of $\mathrm{KBr} \cdot \mathrm{K}^{+}$, can be used for semiquantitative characterisation. As to the anions, molecular ion and monomeric adduct ion intensity ratios reveal to be suitable. The R.S.D. on the relative peak intensities is typically $2-5 \%$. The inherent instrumental precision and accuracy of peak ratio measurements has been assessed on natural isotope contributions and reveals to be within $2 \%$ for signals of at least 500 counts per analysis time. There are no systematic deviations as encountered for intense peaks in TOF S-SIMS. On the other hand, counting errors of up to $13 \%$ occur for low intensity signals. The precision of peak intensity ratios for different ions is typically $5-10 \%$ and improves with intensity. The relative intensities of signals from different analytes in mixtures are similar and result in an estimated uncertainty of $10 \%$ for concentrations derived from the empirical calibration.

Comparison of the calibration for the positive ions in TQ and TOF S-SIMS shows that the $\mathrm{NaBr} \cdot \mathrm{Na}^{+} / \mathrm{KBr} \cdot \mathrm{K}^{+}$ratio can be equally well used as that of $\mathrm{NaBr} \cdot \mathrm{Na}^{+} / \mathrm{KBr} \cdot \mathrm{K}^{+}$in the case of TQ S-SIMS and freeze dried mixtures. This has not been the case for TOF S-SIMS unless melts have been used. The improved sample preparation at INEEL as well as the larger impact area of $\mathrm{ReO}_{4}{ }^{-}$in TQ S-SIMS explains this observation. An additional point of interest is the dynamic range of the response relative to the R.S.D., which determines the range of concentrations that can be distinguished. In this respect, $\mathrm{ReO}_{4}{ }^{-}$-TQ S-SIMS and $\mathrm{Ga}^{+}$-TOF S-SIMS perform comparably. As to the anions, the calibration of the relative peak intensities for $\mathrm{NaBr}$ over $\mathrm{KBr}$ is essentially similar to that obtained for $\mathrm{Ga}^{+}-\mathrm{TOF}$ S-SIMS.

\subsection{Calibration of IT S-SIMS with freeze-dried mixtures}

Here the defocused beam of $\mathrm{ReO}_{4}{ }^{-}$polyatomic primary ions is used as in TQ S-SIMS but the time-domain of ion collection and mass analysis as well as the sensitivity to space charge effects are distinctive features between IT and TQ SSIMS. Specifically, the typical deviation between the experimental and expected isotopic abundance remains within 2-3\% for peaks about 300 and 30,000 counts per analysis time on the condition that the dynamic range to covered remains within a factor of 3 . Space charge effects are believed to be responsible for the loss of precision and accuracy but can be alleviated by ejection of the atomic ions. Typical R.S.D. values are in the order of $15 \%$ for adduct ion intensity ratios measured within the same day on different samples.

The experimental calibration has been found to work out satisfactorily with freeze-dried mixtures using the peak intensity ratios of $\mathrm{NaBr} \cdot \mathrm{K}^{+}$and $\mathrm{KBr} \cdot \mathrm{K}^{+}$over $\mathrm{NaBr} \cdot \mathrm{Na}^{+}$for the positive ions. Fig. 9 shows that the dynamic range is comparable to that in TQ S-SIMS. The relative intensities of molecular anion and negative adduct ions consistently reflect 


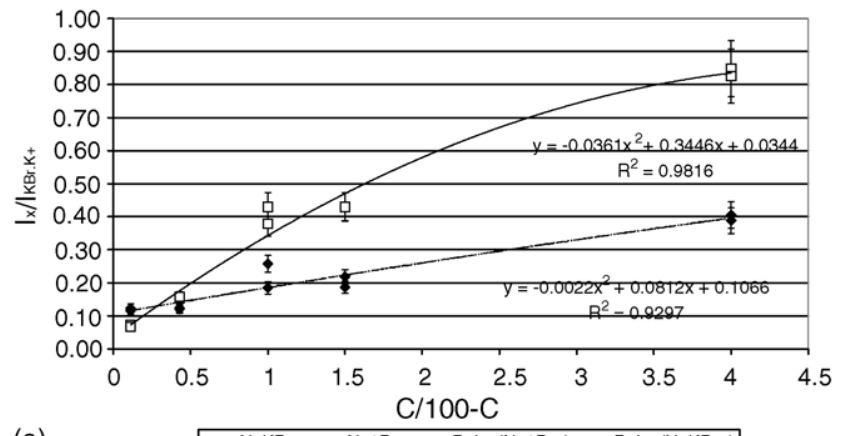

(a)

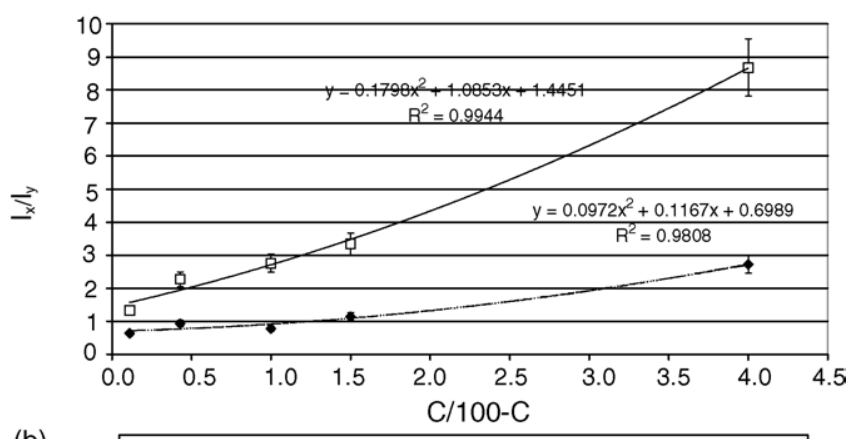

(b)

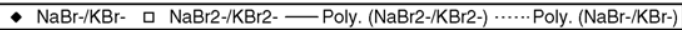

Fig. 9. Calibration of specific positive (a) and negative (b) ion intensity ratios as a function of the $\mathrm{NaBr}$ concentration in a $\mathrm{NaBr}-\mathrm{KBr}$ mixture using freeze-dried samples recorded with IT S-SIMS.

the local concentration. Also here, the peak intensity ratios plotted as a function of the converted variable $X$ gives almost linear relationships as in the case of TQ-S-SIMS. The sensitivity to the number of ions in the IT does not really reduce the potential of IT S-SIMS for the quantitative characterisation. The uncertainty on the local concentration is to be estimated around $10 \%$.

\section{Conclusions}

It has been demonstrated that fast freezing and cryolyophilisation of solutions provides an adequate means to prepare suitable reference mixtures of binary samples for the empirical calibration of the intensities of molecule-specific ions in S-SIMS as a function of the local content. The applicability of the methodology has been verified in two different laboratories and in combination with different S-SIMS instruments, including a TOF S-SIMS with $\mathrm{Ga}^{+}$primary ions, an ion trap and a triple quadrupole S-SIMS, both being equipped with a gun for $\mathrm{ReO}_{4}{ }^{-}$polyatomic projectiles. The use of a defocused primary ion beam and/or bombardment with polyatomic instead of monoatomic primary ions has been found to be beneficial for the semi-characterisation of local mixtures. An indication in the same direction is found by the improved capabilities of TOF S-SIMS when reference mixtures are prepared from melts instead of cryo-lyophilised solutions. However, the experimental uncertainty of $10 \%$ for the concentrations derived from the empirical calibration is considered as acceptable for many material science applications. To the best of our knowledge, this study has been the first one to use molecule-specific high $\mathrm{m} / \mathrm{z}$ ions for the semi-quantitative characterisation of inorganic salts in ultimate surface layers of micro-particles. The preparation of the reference mixtures remains the main bottleneck to push the limits further and extend the methodology to the quantification of more complicated multi-component systems with improved accuracy.

\section{Acknowledgements}

This work was supported in part by the Belgian Office for Scientific, Technical and Cultural Affairs (IUAP 5) and by FWO, Brussels, Belgium (research projects G.0090.98 and G.0172.00). R. Van Ham is indebted to the FWO for a travel grant to INEEL.

\section{References}

[1] J. Vickerman, D. Briggs (Eds.), TOF-SIMS: Surface Analysis by Mass Spectrometry, IM Publications and Surface Spectra Limited, Chichester and Manchester, 2001, p. 789.

[2] R. Van Ham, L. Van Vaeck, F. Adams, A. Adriaens, Anal. Chem. 76 (9) (2004) 2609.

[3] R. Van Ham, L. Van Vaeck, F. Adams, A. Adriaens, Anal. Chim. Acta 500 (1-2) (2003) 259.

[4] V. Ignatova, L. Van Vaeck, R. Gijbels, F. Adams, Int. J. Mass Spectrom. 225 (3) (2003) 213.

[5] V. Ignatova, L. Van Vaeck, R. Gijbels, F. Adams, Vacuum 69 (1-3) (2002) 307.

[6] A. Benninghoven, in: J. Vickerman, D. Briggs (Eds.), TOF-SIMS: Surface Analysis by Mass Spectrometry, IM Publications and Surface Spectra Limited, Chichester and Manchester, 2001, p. 41.

[7] J.E. Delmore, A.D. Appelhans, E.S. Peterson, Int. J. Mass Spectrom Ion Process. 146/147 (1995) 15.

[8] R. Van Ham, A. Adriaens, L. Van Vaeck, F. Adams, J. Aerosol. Sci. 31 (2002) S396.

[9] V. Ignatova, L. Van Vaeck, R. Van Ham, A. Adriaens, F. Adams, Nucl. Instr. Meth. Phys. Res. A 480 (2002) 54-64.

[10] A. Croisy, J.L. Guerquin-Kern, Biofutur 175 (1998) A2.

[11] F. Honda, Y. Fuyuka, J.W. Rabalais, J. Chem. Phys. 70 (1979) 4834.

[12] R. Van Ham, L. Van Vaeck, A. Adriaens, F. Adams, B. Hodges, G. Groenewold, J. Anal. Atom. Spectrosc. 17 (2002) 753.

[13] R. Van Ham, Molecular Speciation Using Static Secondary Ion Mass Spectrometry (S-SIMS): Methodology and Applications Dissertation, University of Antwerp, Belgium, 2002. 